\title{
Editorial: Big Data and Cyber-Physical-Social Computing
}

\author{
Song Guo ${ }^{1} \cdot$ Zhen $\mathrm{Liu}^{2} \cdot$ Peng $\mathrm{Li}^{3}$ \\ Published online: 3 July 2019 \\ (C) Springer Science+Business Media, LLC, part of Springer Nature 2019
}

\section{Editorial:}

Data is becoming an increasingly decisive resource in modern societies, economies, and governmental organizations. Big Data is an emerging paradigm encompassing various kinds of complex and large scale information beyond the processing capability of conventional software and databases. CyberPhysical-Social Computing (CPSC) is characterized by the deep complex intertwining among cyber/physical components and integrating Human into Computing. CPSC encompasses not only cyberspace and physical world, but also human knowledge, mental capacity, and sociocultural elements. This nature of "big data" imposes fundamental challenges on the design and management of CPSC in multiple aspects such as performance, energy efficiency, security, privacy, reliability, sustainability, fault tolerance, scalability and flexibility. Tackling these challenges necessitates innovative big data techniques for handling massive data in CPSC. This special issue presents the state-of-the-art research results on the topic of big data sensing, processing and storage for CPSC and enabling technologies in developing and optimizing the architecture, design, and operation of CPSC.

This special issue features five selected papers with high quality. The first article, "RLPAS: Reinforcement Learningbased Proactive Auto-Scaler for Resource Provisioning in Cloud Environment", authored by Bibal Benifa, propose the RLPAS (Reinforcement Learning based Proactive AutoScaler) algorithm that allocates necessary resources by learning the environment dynamically. It outperforms existing auto-scaling approaches in terms of CPU utilization, response time and throughput.

Peng Li

pengli@u-aizu.ac.jp

1 Department of Computing, The Hong Kong Polytechnic University, Hung Hom, Hong Kong

2 Computational Intelligence Laboratory, Nagasaki Institute of Applied Science, Nagasaki, Japan

3 University of Aizu, Aizu, Japan

The second article titled "Green Massive Traffic Offloading for Cyber-Physical Systems over Heterogeneous Cellular Networks" presents an analysis on CPS offloading rate and achievable throughput when small cells base stations (SCBSs) are powered by solar energy. The solar energy harvesting allows SCBSs to offset the costs of serving CPS devices.

In the next article with the title "Local Core Members Aided Community Structure Detection", the authors propose an effective method in detecting community structure in social networks based on the closeness of relations among neighbors. This method calculate the gravity between each neighbor node to core nodes, then makes judgement if the node should be classified in the community or not, and finally form the process of community detection.

The fourth article titled "A Prediction Model of the Project Life-span in Open Source Software Ecosystem" provides an insight on the project life-span for a free open source software ecosystem. The statistical analysis of some project characteristics in GitHub is presented, and authors find that the choices of programming languages, the number of files, the label format of the project, and the relevant membership expressions can impact the life-span of a project. Based on these discovered characteristics, authors also propose a prediction model to estimate the project life-span in open source software ecosystems. These results may help developers reschedule the project in open source software ecosystem.

The last article titled "Exploring Temporal Analytics in Fog-Cloud Architecture for Smart Office Healthcare" investigates utilizing the potential of IoT Technology to provide a novel Fog-Cloud architecture for efficient healthcare services in smart office. Specifically, a FogCloud architecture has been proposed to monitor and analyze various health attributes of a person during his working hours. Moreover, the framework indulges various activities in the ambient office environment with the purpose of analyzing it for health severity. In order to realize this, a probabilistic measure, named as Severity Index (SI) is defined to evaluate the adverse effects of different activities on personal health. Finally, an application scenario 
of temporal healthcare predictive monitoring and alert generation is discussed to depict the ideology of Smart Office Healthcare.

Acknowledgements The guest editors are thankful to our reviewers for their effort in reviewing the manuscripts. We also thank the Editin-Chief, Dr. Imrich Chlamtac for his supportive guidance during the entire process.

Publisher's Note Springer Nature remains neutral with regard to jurisdictional claims in published maps and institutional affiliations.

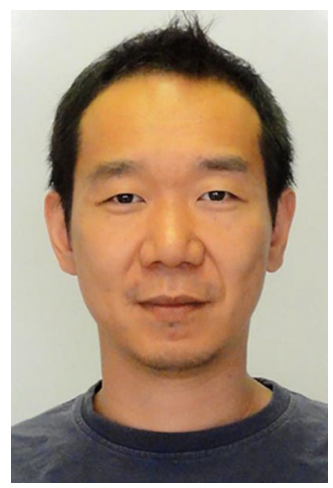

Song Guo is a Full Professor at Department of Computing, The Hong Kong Polytechnic University. His research interests are mainly in the areas of big data, cloud computing, mobile computing, and distributed systems with over 450 papers published in major conferences and journals. His work was recognized by the 2016 Annual Best of Computing: Notable Books and Articles in Computing in ACM Computing Reviews. He is the recipient of the 2018 IEEE TCGCC Best Magazine Paper Award, 2017 IEEE Systems Journal Annual Best Paper Award, and other six Best Paper Awards from IEEE/ACM conferences. Prof. Guo was an Associate Editor of IEEE Transactions on Parallel and Distributed Systems and an IEEE ComSoc Distinguished Lecturer. He is now an Associate Editor of IEEE Transactions on Cloud Computing, IEEE Transactions on Emerging Topics in Computing, IEEE Transactions on Sustainable Computing, IEEE Transactions on Green Communications and Networking, and IEEE Network. Prof. Guo also served as General and Program Chair for numerous IEEE conferences. He currently serves as a Director and Member of the Board of Governors of IEEE ComSoc.

Zhen Liu is a professor of Graduate School of Engineering, director of Computational Intelligence Laboratory, Nagasaki Institute of Applied Science, Japan. He received his Ph.D. degree in information science from Tohoku University. He has been working on database and computational intelligence. His research interests include big data and data mining, decision making and DSS, high performance computing, and assistive engineering. He is a member of ACM, IEEE, JSAI, IPSJ, IEICE.

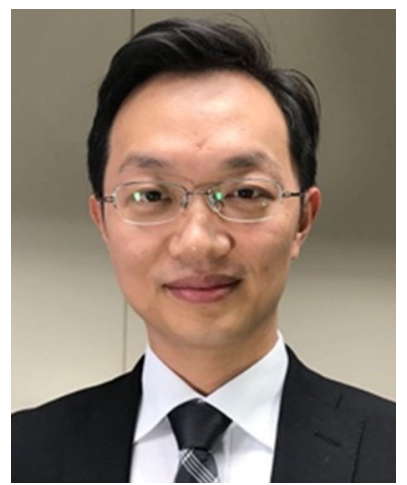

Peng Li received his BS degree from Huazhong University of Science and Technology, China, in 2007, the MS and PhD degrees from the University of Aizu, Japan, in 2009 and 2012, respectively. Dr. Li is currently an Associate Professor in the University of Aizu, Japan. His research interests mainly focus on cloud computing, Internet-ofThings, big data systems, as well as related wired and wireless networking problems. He won the Young Author Award of IEEE Computer Society Japan Chapter in 2014. He won the Best Paper Award of IEEE TrustCom 2016. He supervised students to win the First Prize of IEEE ComSoc Student Competition in 2016. He is a member of IEEE. 